\title{
Structural insights into the negative regulation of BRI1 signaling by BRI1-interacting protein BKI1
}

Jie Wang ${ }^{1, *}$, Jianjun Jiang ${ }^{2, *}$, Jue Wang ${ }^{1, *}$, Lei Chen ${ }^{1}$, Shi-Long Fan ${ }^{1}$, Jia-Wei Wu ${ }^{1}$, Xuelu Wang ${ }^{2,3}$, Zhi-Xin Wang ${ }^{1}$

${ }^{1}$ MOE Key Laboratory for Protein Science, School of Life Sciences, Tsinghua University, Beijing 100084, China; ${ }^{2}$ State Key Laboratory of Genetic Engineering and Institute of Plant Biology, School of Life Sciences, Fudan University, Shanghai 200433, China; ${ }^{3}$ College of Life Science and Technology, Huazhong Agricultural University, Wuhan, Hubei 430070, China

Brassinosteroids (BRs) are essential steroid hormones that have crucial roles in plant growth and development. BRs are perceived by the cell-surface receptor-like kinase brassinosteroid insensitive 1 (BRI1). In the absence of BRs, the cytosolic kinase domain (KD) of BRI1 is inhibited by its auto-inhibitory carboxyl terminus, as well as by interacting with an inhibitor protein, BRI1 kinase inhibitor 1 (BKI1). How BR binding to the extracellular domain of BRI1 leads to activation of the KD and dissociation of BKI1 into the cytosol remains unclear. Here we report the crystal structure of BRI1 KD in complex with the interacting peptide derived from BKI1. We also provide biochemical evidence that BRI1-associated kinase 1 (BAK1) plays an essential role in initiating BR signaling. Steroiddependent heterodimerization of BRI1 and BAK1 ectodomains brings their cytoplasmic KDs in the right orientation for competing with BKI1 and transphosphorylation.

Keywords: BR signaling; BKI1 inhibition; BRI1 activation; BRI1-BAK1 transphosphorylation

Cell Research (2014) 24:1328-1341. doi:10.1038/cr.2014.132; published online 21 October 2014

\section{Introduction}

Signal perception through cell surface receptors, and transduction of this signal to the cell's interior, are essential to all life forms [1]. In plants, a large family of such receptors is the leucine-rich repeat receptor-like kinases (LRR-RLKs), with more than 220 members in Arabidopsis and about 400 in rice. Each LRR-RLK contains a leucine-rich repeat extracellular domain that usually binds ligands, a single membrane-spanning helix, and a cytoplasmic kinase domain that initiates intracellular signal

\footnotetext{
*These three authors contributed equally to this work.

Correspondence: Zhi-Xin Wang

E-mail: zhixinwang@mail.tsinghua.edu.cn

Abbreviations: BRs (brassinosteroids); BRI1 (brassinosteroid insensitive 1); BKI1 (BRI1 kinase inhibitor 1); BAK1 (BRI1-associated kinase 1); LRRRLKs (leucine-rich repeat receptor-like kinases); BIM (BRI1-interacting motif); CDs (cytosolic domains); KD (kinase domains); Cter (C-terminal); BSKs (BR-signaling kinases); JM (juxtamembrane); rmsd (root-meansquare deviation); PKA (protein kinase A); MAKR (membrane associated kinase regulators); PM (plasma membrane); pBKI1-Cter (phosphorylated BKI1-Cter)

Received 26 June 2014; revised 4 September 2014; accepted 10 September 2014; published online 21 October 2014
}

transduction [2]. Two of the well-studied LRR-RLKs are brassinosteroid-insensitive 1 (BRI1), the brassinosteroid (BR) receptor, and BRI1-associated kinase 1 (BAK1) that serves as a coreceptor for BRI1 activation [3-5]. BRs are plant-specific steroids that play essential roles in a wide range of developmental and physiological processes, such as stem elongation, leaf development, vascular differentiation, male fertility, flowering time, senescence, stomata development, and resistance to biotic and abiotic stresses [6-9]. Mutations of BRI1 or other components of the BR biosynthetic or signaling pathway cause severe growth abnormalities, including dwarfism, male sterility, delayed flowering, reduced apical dominance and photomorphogenesis in darkness. In contrast, increased BR content or enhanced BR signaling can enhance cellulose biosynthesis, biomass accumulation, and plant growth.

BR signaling is initiated by BR binding to a cell surface receptor kinase, BRI1 [10-12]. In the absence of BRs, the BRI1 KD is kept in a basal state by its auto-inhibitory carboxyl terminus [13] and by interacting with an inhibitor protein BKI1 [14]. BR binding to the extracellular domain of BRI1 causes autophosphorylation of the BRI1 KD, release of BRI1 C-terminal autoinhibition, 
and BKI1 phosphorylation and its dissociation from the plasma membrane, leading to the formation of BRI1 and BAK1 complex [13, 15-17]. The KDs of BRI1 and BAK1 transphosphorylate each other on multiple sites to form fully activated receptor [17]. The activated BRI1 can phosphorylate several receptor-like cytoplasmic kinases, including the BR-signaling kinases (BSKs) and constitutive differential growth 1 , which interact with and presumably activate the bril-suppressor 1 phosphatase to transduce BR signaling downstream of BRI1 [18, 19]. The BSK family includes 12 members (BSK1-12) which play redundant roles in BR signaling [20].

Although the activation of BR signaling has been extensively studied during the past decade, a major gap in our understanding lies in the early molecular events that regulate BR-induced BRI1/BAK1 association and reciprocal phosphorylation. How BR binding to BRI1 ectodomain leads to partial activation or autophosphorylation of BRI1 KD has remained unclear. In the present study, we report the crystal structure of BRI1 $\mathrm{KD}$ in complex with the BRI1-interacting motif derived from BKI1. We also provide clear biochemical evidence that BAK1 plays an essential role in the early events of BRI1 activation, and BKI1 competitively inhibits transphosphorylation on the cytosolic domains of BAK1 and BRI1. Steroid-induced heterodimerization of BRI1 and BAK1 ectodomains brings their cytoplasmic KDs in the right orientation for competing with BKI1 and transphosphorylation. These results not only reveal the structural basis underlying BKI1 recognition by BRI1, but also provide insight into the initiating mechanism of brassinosteroid-induced BRI1 activation.

\section{Results}

Biochemical characteristics of the cytosolic domains of $B R I 1$ and BAKI

To understand how BKI1 negatively regulates BRI1 signaling, we expressed and purified the cytosolic domains of BRI1 (BRI1-CD, residues 815-1196) and BAK1 (BAK1-CD, residues 255-615), the KD of BSK3 (BSK3-KD, residues 36-329), and the C-terminal region of BKI1 (BKI1-Cter, residues 257-337; Figure 1A). Bacterially expressed BRI1 and BAK1 proteins are catalytically active, because they undergo autophosphorylations during the expression and purification procedure $[17$, 21]. Western blotting confirmed that the recombinant BRI1-CD and BAK1-CD are phosphorylated on multiple sites when purified from E. coli (Figure 1B). The phosphorylated proteins can be dephosphorylated by a widely used protein phosphatase PP2Ca, and the corresponding kinase-dead mutants, BRI1-CD ${ }^{\mathrm{K} 911 \mathrm{R}}$ (mBRI1-CD) and
BAK1-CD ${ }^{\text {D434N }}$ (mBAK1-CD), are completely unphosphorylated (Figure 1B). The purified mBAK1-KD, but not mBRI1-KD, can be rapidly phosphorylated by BRI1$\mathrm{CD}$ in the presence of $1 \mathrm{mM}$ ATP and $10 \mathrm{mM} \mathrm{Mg}^{2+}$ [21]. In contrast, mBRI1-CD, rather than mBAK1-CD, can be phosphorylated by BAK1-CD (Figure 1C). The inability of an active BRI1-CD to transphosphorylate an inactive mutant mBRI1-CD suggests that the mechanism of autophosphorylation is intramolecular or mediated by the nascent kinase passing through a transitory intermediate form [22].

To characterize the steady-state kinetic properties of BRI1-CD, the enzyme-catalyzed phosphorylation reaction was monitored using an enzyme-coupled spectrophotometric assay that couples production of ADP to oxidation of NADH, measured as a decrease in absorbance at $340 \mathrm{~nm}$ [23]. Figure 1D shows a typical progress curve of the BRI1-catalyzed BSK3-KD phosphorylation. When all of the substrates have been converted to products, the phosphorylation stoichiometry of BSK3-KD was determined to be close to $1 \mathrm{~mol}$ of phosphate/mol of BSK3KD (Supplementary information, Figure S1), consistent with the previous findings that BRI1 specifically phosphorylated BSK3-KD at Ser212 within the BRI1 consensus phosphorylation sequence $\left({ }^{206}{ }^{R D G K S Y S T N L A F T ~}{ }^{218}\right)$ both in vitro and in vivo [18]. The BRI1-CD-catalyzed reaction obeyed classical Michaelis-Menten kinetics (inset of Figure 1D). Direct curve fitting of the data to the Michaelis-Menten equation yielded $k_{\text {cat }}$ and $K_{\mathrm{m}}$ values, $3.99 \pm 0.21 \mathrm{~s}^{-1}$ and $20.97 \pm 3.30 \mu \mathrm{M}$, respectively, indicating that BSK3 is an efficient substrate for BRI1.

BKI1 negatively regulates BR signaling through two evolutionarily conserved motifs: a lysine/arginine-rich motif that targets BKI1 to the plasma membrane, and a BRI1-interacting motif (BIM, residues 306-325) that binds to the BRI1 KD. There are two phosphorylation sites, Ser270 and Ser274, in BKI1-Cter, which play important roles in subsequent phosphorylation of Tyr211 and dissociation of BKI1 from the plasma membrane [14, $15,19]$. In vitro kinase assay confirmed that the purified BKI1-Cter can be rapidly phosphorylated by BRI1-CD (Figure 1E). Inset of this figure shows the variation of initial rates of the BRI1-catalyzed reaction with BKI1Cter concentrations. By fitting the Michaelis-Menten equation to the experimental data, the values of $k_{\text {cat }}$ and $K_{\mathrm{m}}$ were determined to be $0.97 \pm 0.06 \mathrm{~s}^{-1}$ and $4.28 \pm 0.73$ $\mu \mathrm{M}$, respectively. Both the $k_{\text {cat }}$ and $K_{\mathrm{m}}$ values decreased about fourfold as compared with those of BSK3-KD substrate, suggesting that the two substrates may have distinct interaction modes with BRI1-CD. The substrate specificity constant $k_{\text {cat }} / K_{\mathrm{m}}$ for BRI1-catalyzed BKI1-Cter phosphorylation was $2.3 \times 10^{5} \mathrm{M}^{-1} \mathrm{~s}^{-1}$, which is similar 

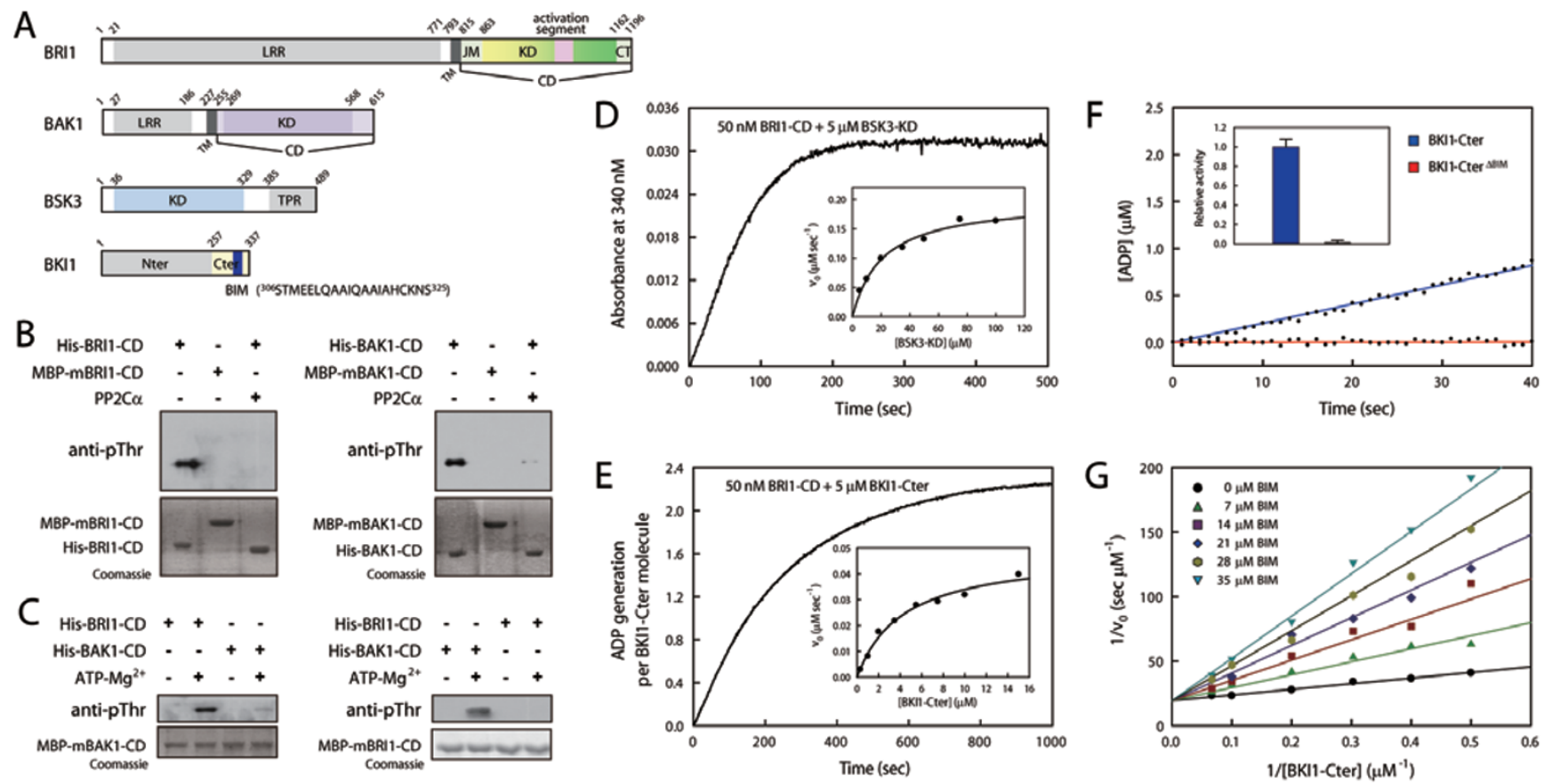

Figure $1 \mathrm{BIM}$ is a major determinant for BRI1-catalyzed BKI1 phosphorylation. (A) Schematic diagram of BRI1, BAK1, BSK3 and BKI1. LRR, leucine-rich repeat domain; TM, transmembrane domain; CD, cytosolic domain; KD, kinase domain; TPR, tetratricopeptide repeat domain; Nter, N-terminal domain; Cter, C-terminal domain; BIM, BRI1-interacting motif. The sequence of the BIM peptide (blue) is provided. (B) Phosphorylation states of recombinant wild-type, kinase-dead mutant and PP2Ca-dephosphorylated BRI1-CD and BAK1-CD analyzed using a general anti-pThr antibody. (C) Transphosphorylation analyses of BRI1-CD and BAK1-CD by western blotting. (D and E) Representative time course of BRI1-CD-catalyzed phosphorylation on BSK3-KD (D) and BKI1-Cter (E). The insets shows the initial rates vs the BSK3-KD and BKI1-Cter concentrations, respectively. (F) Time courses of BRI1-CD-catalyzed phosphorylation of BKI1-Cter and BKI1-Cter ${ }^{\triangle B I M}(5 \mu M)$. The relative activities of BRI1-CD towards two substrates (mean $\pm \mathrm{SEM}, n=3$ ) are shown in the inset. (G) Competitive inhibition of BRI1-catalyzed BKI1-Cter phosphorylation by BKI1-BIM. The kinetic parameters were obtained by fitting the experimental data to the competitive inhibition equation. See also Supplementary information, Data S1 and Figure S1.

to that of the BRI1-catalyzed BSK3-KD phosphorylation $\left(1.9 \times 10^{5} \mathrm{M}^{-1} \mathrm{~s}^{-1}\right)$. In comparison, neither BSK3-KD nor BKI1-Cter could be effectively phosphorylated by BAK1-CD under the same experimental conditions.

To quantitatively assess the contribution of the BIM peptide to the BRI1-catalyzed reaction, we measured the phosphorylation efficiency of BKI1-Cter ${ }^{\mathrm{BIIM}}$ (a BIM-deleted BKI1-Cter mutant) by BRI1-CD. As shown in Figure 1F, compared with intact BKI1-Cter, deletion of the BIM region resulted in a dramatic drop (more than 80 -fold) of the phosphorylation rate, suggesting that the phosphoacceptor sequence of BKI1-Cter in itself binds weakly to the active site of BRI1-CD, and the BRI1-interacting motif is a major determinant for the substrate specificity of BKI1-Cter [15, 19]. We also investigated the mechanism by which a synthetic BIM peptide ${ }^{306} \mathrm{ST}$ MEELQAAIQAAIAHCKNS ${ }^{325}$ ) inhibits BRI1 kinase activity towards BKI1-Cter substrate. The kinetics of the BRI1-CD-catalyzed phosphorylation of BKI1-Cter were examined in the presence of various fixed concentrations of BIM peptide. Double-reciprocal plots of initial rate data over a range of concentrations for both the BIM peptide and BKI1-Cter substrate revealed a simple competitive pattern with a $K_{\mathrm{i}}$ value of $5.40 \pm 0.90 \mu \mathrm{M}$ (Figure 1G), which is comparable to the $K_{\mathrm{m}}$ value for the BRI1-CD-catalyzed BKI1-Cter phosphorylation. This result further confirms that the BIM peptide was sufficient to tightly interact with the BRI1 KD [15].

\section{Crystal structure of BRII KD in complex with BIM pep- tide}

First, we carried out kinase activity assays with three fragments of the BRI1 cytoplasmic domain to assess the roles of the juxtamembrane (JM) and Cter regions (Supplementary information, Table S1). The JM region, KD and Cter segment have been previously assigned using a BRI1 homology model [15]. In the presence of the JM region, the $K_{\mathrm{m}}$ values of the fragments containing the JM 
and/or Cter regions are only slightly lower than that of the KD alone. Our results are in contrast to those of $\mathrm{Oh}$ et al. [24] who reported that deletion of JM residues up to 882 caused a severe reduction in BRI1 kinase activity. One possible explanation for this discrepancy between the two studies might be that the construct of BRI1 KD used in their study was not fully active since deletion of residues 863-882 (the $\alpha \mathrm{B}$ helix in the BRI1-KD structure) has a strong impact on solubility of the construct.

To gain a structural understanding of the mechanism of BKI1 inhibition, we systematically screened for crystallizable fragments of the BRI1 cytoplasmic domain and obtained crystals of BRI1-KD (863-1162) in complex with the BKI1-BIM peptide and AMP-PNP. The crystal structure of the ternary complex was determined at 2.2 $\AA$ resolution (Table 1). It should be indicated that the JM region is crucial for in vivo BRI1 function [13, 24, 25], and thus some aspects of BRI1's physiological function cannot be fully elucidated using our structural model. The crystallographic asymmetric unit contains two mol-

Table 1 The structural and refinement statistics for the BRI1-KD-BIM complex

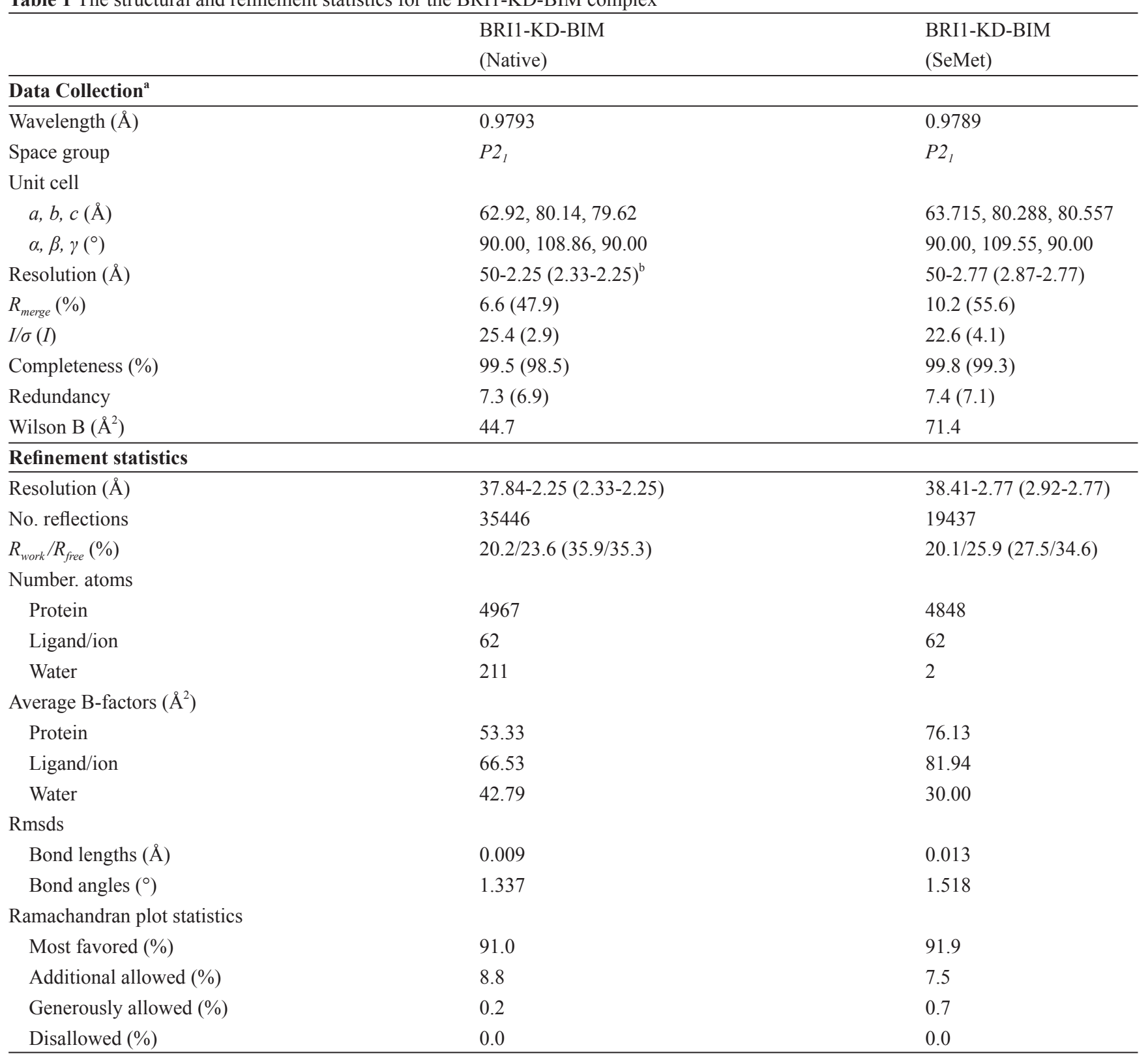

${ }^{a}$ All data sets were collected from a single crystal.

${ }^{b}$ Values in the parentheses are for the highest-resolution shell. 
ecules (A and B) of BRI1-KD-BIM complex. In both A and $\mathrm{B}$ molecules, 16 of the BIM residues $\left({ }^{307}\right.$ TMEELQAAIQAAIAHC ${ }^{322}$ ) are clearly visible in the electron-density map. The overall structures of BRI1-KD in molecules $\mathrm{A}$ and $\mathrm{B}$ are similar, and the two structures overlap with one another with a root-mean-square deviation (rmsd) between all $\mathrm{C} \alpha$ atoms of $0.47 \AA$ (Supplementary information, Figure S2A and S2B). The structural analyses hereafter are on the basis of molecule A. In the BRI1KD-BIM complex, the catalytic domain of BRI1 displays the typical protein kinase fold which contains a small $\mathrm{N}$-terminal lobe comprised mainly of $\beta$ strands, and a large C-terminal lobe that consists mostly of $\alpha$ helices (Figure 2A). The BIM peptide adopts a helical conformation, as confirmed by the well-defined electron density (Supplementary information, Figure S2C), and binds to a groove present between $\alpha \mathrm{H}$ and $\alpha \mathrm{G}$ in the C-lobe of
BRI1-KD, located distal to the common substrate-binding region. Topologically, the structure of BRI1-KD is similar to that of the active BAK1-KD (PDB ID: 3UIM) [26], with which it shares approximately $40 \%$ sequence identity. The overall rmsd of superposition of BRI1-KD with BAK1-KD is $1.04 \AA$ (Figure 2B and Supplementary information, Figure S2E). A sequence alignment based on the structure is presented in Supplementary information, Figure S2D. Differences only occur in particular amino acids, which may determine their distinct function in various signaling pathways (see later).

The activation segment in BRI1-KD (residues Asp1027-Glu1056) is well-ordered and has the conformation typical of all active protein kinases. The structure reveals phosphorylation on the side chains of Thr1039, Ser1042, Ser1044 and Ser1060 in the activation segment of BRI1-KD. The phosphate group of the phosphorylat-
A

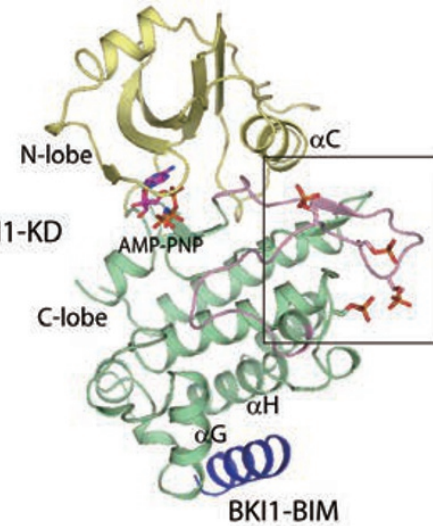

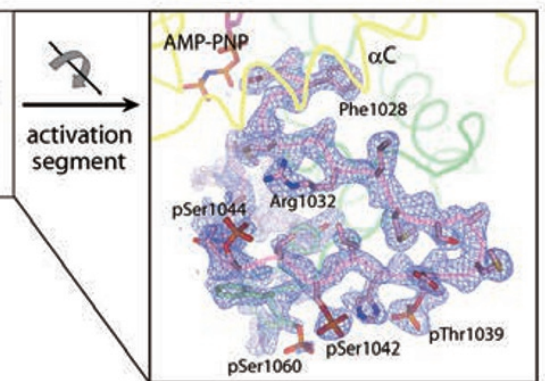

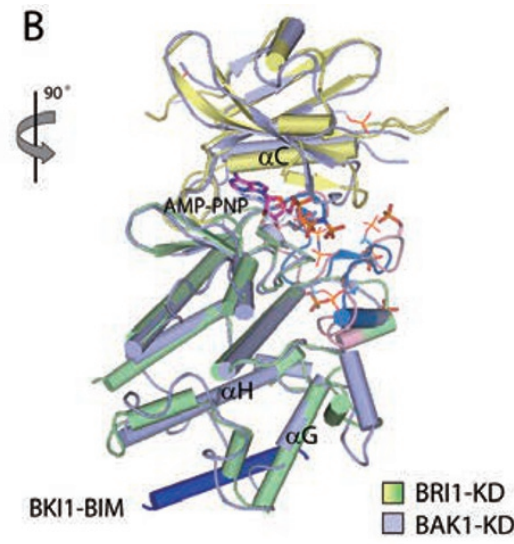

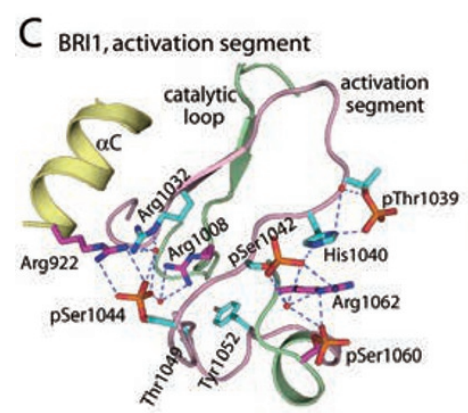
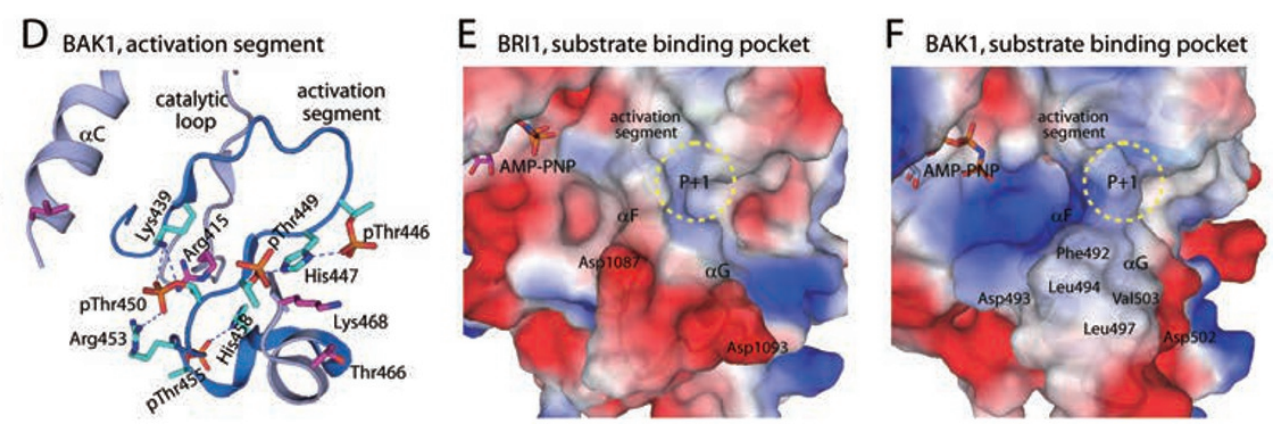

Figure 2 Overall structure of BRI1-KD in complex with BKI1-BIM. (A) Ribbon diagram of BRI1-KD-BIM complex. The N-lobe, $\mathrm{C}$-lobe, and the activation segment of BRI1-KD are colored in pale yellow, pale green and pink, respectively, and BKI1-BIM is shown in blue. AMP-PNP and the observed phosphorylated residues are shown as sticks. The coloring scheme of BRI1-KD is the same in the following figures unless indicated. The inset shows the $F_{\mathrm{o}}-F_{\mathrm{c}}$ omit map (contoured at $2.0 \sigma$ ) for the activation segment of BRI1-KD. (B) Comparison of BRI1-KD and BAK1-KD. BAK1-KD is colored in light blue with its activation segment in marine blue, and its phosphorylated residues and bound AMP-PNP are shown as lines. (C and D) Interaction networks involving phosphor-Thr/Ser residues in the activation segment of BRI1 (C) and BAK1 (D). Residues in the activation segments are highlighted as cyan sticks. Blue dashed lines represent the polar interactions. (E and $\mathbf{F}$ ) Molecular determinants for the substrate specificity of BRI1 (E) and BAK1 (F). The classic substrate-binding site are encircled by yellow dashed lines. Key residues potentially determining the substrate specificity of BRI1 and BAK1 are indicated. See also Supplementary information, Figure S2 and Table S1. 
ed Ser1044 is positioned to compensate for a positively charged pocket, which is clustered by three arginine residues Arg922, Arg1008 and Arg1032 (Figure 2C and 2D). Residue Ser1044 is the key phosphorylation site corresponding to Thr197 in protein kinase A (PKA), whose phosphorylation is important for promoting the correct orientation and electrostatic environment for the catalytic base Asp1009, as well as the proper conformation of helix $\alpha \mathrm{C}$. Overexpression of BRI1 ${ }^{\mathrm{S} 1044 \mathrm{~A}}$ in bri15 transgenic plants failed to rescue the dwarf phenotype [27], indicating that this residue is important for the activation of BRI1. The other three phosphorylated residues form multiple direct and water-mediated interactions with His1040 and Arg1062. Together, massive networks of hydrophilic interactions stabilize the conformation of the activation segment. The AMP-PNP molecule is well positioned in the ATP-binding site formed by the hinge region, the flexible P-loop in the N-lobe, and the DFG motif in the C-lobe (Supplementary information, Figure $\mathrm{S} 2 \mathrm{~F}$ ). There is no density for $\mathrm{Mg}^{2+}$ in the structure although the crystallization condition included sufficient amounts of magnesium. One possible explanation for this is that our crystallization buffer contains $200 \mathrm{mM}$ of citrate which can combine with $\mathrm{Mg}^{2+}$ and greatly reduces the concentration of free $\mathrm{Mg}^{2+}$ in the crystallization buffer. Similar results have been observed in the crystal structures of other kinases, indicating that the absence of the density for magnesium occurs occasionally for kinase structures [26].

BRI1-KD and BAK1-KD recognize distinct target sequences, and therefore exhibit different substrate specificities. BRI1 is a basophilic serine/threonine kinase that preferentially phosphorylates substrates with basic residues in close proximity to the phosphorylation site, and optimum phosphorylation peptide of BRI1-KD required Arg or Lys residues at $\mathrm{P}-3, \mathrm{P}-4$ and $\mathrm{P}+5$ [21]. To provide a structural explanation for substrate selectivity, we compared the surface electrostatic potential of the substrate-binding pockets on BRI1-KD and BAK1-KD (Figure $2 \mathrm{E}$ and $2 \mathrm{~F}$ ). The $\mathrm{P}+1$ specificity site of protein kinases, originally named for its role in contacting the $\mathrm{P}+1$ residue in structure of PKA in complex with a peptide inhibitor [28], is formed by a surface loop (residues Thr1045-Glu1056 in BRI1) contiguous with the activation loop (residues Ser1035-Ser1044 in BRI1). The $\mathrm{P}+1$ site of BRI1-KD is encircled by acidic side chains of Asp1087, Asp1093 and probably pSer1060, which is consistent with the singular preference of BRI1 for positively charged residues around the phosphorylation site on the substrate (Figure 2E). In contrast, the corresponding region of BAK1-KD consists of several hydrophobic residues (Phe492, Leu494, Leu497 and Val503), while the acidic Asp493 and Asp502 point in the opposite directions to that of the corresponding Asp1087 and Asp1093 in BRI1-KD (Figure 2F). These subtle structural differences between BRI1-KD and BAK1-KD may determine their distinct functions in various signaling pathways by recognizing different substrates.

\section{Docking interactions between BIM peptide and BRII KD}

Complex formation between BIM and BRI1-KD buries a surface area of $\sim 638 \AA^{2}$. The traceable region of the BIM peptide folds into a four-turn $\alpha$-helix and is embraced by $\alpha \mathrm{G}$ and $\alpha \mathrm{H}$ from BRI1-KD (Figure 3A). The BIM peptide lies in a hydrophobic groove formed by residues Val1100, Lys 1101, Ala1104 and Ile1108 from helix $\mathrm{G}$ and its succeeding loop at one side and residues Ile1125, Leu1128, Gln1129, Leu1131, Val1135 and Leu1138 from helix $\alpha \mathrm{H}$ at the opposite side. Six hydrophobic residues (Met308, Leu311, Ala314, Ile315, Ala318, Ile319) and the methylene group of Gln312 from the BIM peptide are involved in the hydrophobic interaction with BRI1-KD. In addition, Lys1132 from helix $\alpha \mathrm{H}$ of BRI1-KD forms a hydrogen bond with Gln316 in the BIM peptide of BKI1.

To assess the importance of the aforementioned interactions, we generated a series of point mutations on the BKI1-Cter and examined their effect on the Ser270/ Ser274 phosphorylation of BKI1-Cter catalyzed by BRI1-KD (Figure 3B). When the key hydrophobic residues on the BIM sequence were individually replaced by charged residues or Ala (BKI1-Cter ${ }^{\mathrm{M} 308 \mathrm{D}}$, BKI1-Cter ${ }^{\mathrm{L} 311 \mathrm{D}}$, BKI1-Cter ${ }^{\mathrm{I} 15 \mathrm{D}}$, BKI1-Cter ${ }^{\mathrm{A} 318 \mathrm{D}}$ and BKI1-Cter $\left.{ }^{\mathrm{I} 319 \mathrm{~A}}\right)$, their transphosphorylation rates by BRI1-KD showed a 2-6fold decrease. The triple mutations (BKI1-Cter ${ }^{\mathrm{M} 308 \mathrm{D} / \mathrm{A} 314 \mathrm{D} /}$ A318D and BKI1-Cter ${ }^{\mathrm{L} 311 \mathrm{D} / 1315 \mathrm{D} / 1319 \mathrm{~A}}$ ) resulted in a further decrease in BRI1-KD's transphosphorylation activity towards BKI1-Cter, indicating a negatively synergistic effect in BRI1-KD-BKI1-Cter interaction. As the negative controls, we also made a mutant, BKI1-Cter ${ }^{\mathrm{Q} 312 \mathrm{~A}}$, and assessed its effect on phosphorylation rate. As expected, no significant effect was observed for this mutant. Interestingly, the Q316E mutation yielded a modest but marked increase in its phosphorylation rate by BRI1$\mathrm{KD}$, probably because Glu316 in this mutant formed a salt bridge with Lys1132 of BRI1-KD which enhances the BRI1/BKI1 interaction. BKI1 represents a class of membrane associated kinase regulators (MAKRs) with 7 members in Arabidopsis, which contain both the $\mathrm{N}$-terminal and C-terminal linear motifs. Jaillais et al. [15] have shown that some members of MAKR family (MAKR3 and MAKR4) could not interact with BRI1$\mathrm{KD}$ in vitro, and their overexpression did not result in a BR-related phenotype. Secondary structure predictions 

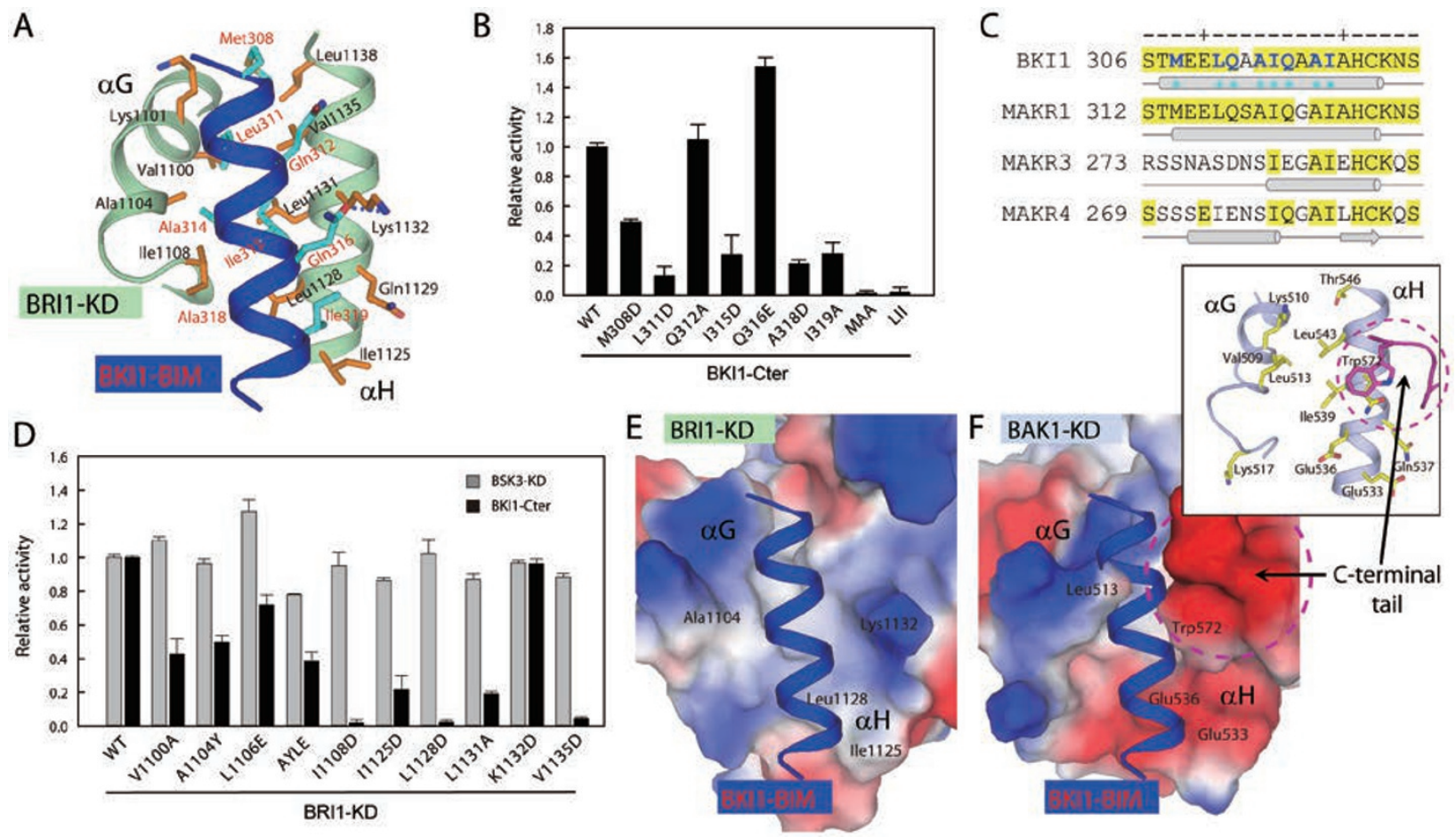

Figure 3 BKI1-BIM specifically recognizes BRI1-KD. (A) Interactions between BKI1-BIM and BRI1-KD. Residues from BKI1$\mathrm{BIM}$ and BRI1-KD are highlighted as cyan and orange sticks, respectively. (B) Effects of BKI1 mutants on the phosphorylation efficiencies of BRI1-KD towards BKI1-Cter fragment (mean \pm SEM, $n=3$ ). (C) Sequence alignment of the BIM regions from BKI1 and MAKRs. The UniProt identifiers for BKI1, MAKR1, MAKR3, and MAKR4 are Q9FMZ0, Q3E936, Q9ZUS8 and O80624, respectively. The key residues required for BRI1-BKI1 recognition are indicated by cyan triangles. The observed and predicted secondary structure assignments are shown below each sequence. (D) Effects of BRI1-KD mutants on its phosphorylation efficiencies towards substrates BKI1-Cter and BSK3-KD (mean \pm SEM, $n=3$ ). (E and F) Comparison of the docking grooves for BKI1-BIM on BRI1-KD (E) and BAK1-KD (F). The BRI1-KD and BAK1-KD are shown in surface representation colored according to electrostatic potential (positive, blue; negative, red), and the BKI1-BIM peptide is highlighted as blue ribboon. The hindrance of Trp572 from the C-terminal tail of BAK1-KD for BKI1-BIM binding is shown in the inset of F.

using the procedure of Rost and Sander [29] delineated residues 273-281 in MAKR3 and 279-288 in MAKR4 as a region of highest non-helix probability (Figure 3C), which indicates that integrity of the $\alpha$-helical structure is also required for BKI1's inhibitory function.

Next, we systematically substituted key residues in the BRI1 interface and determined the contribution of individual residue to the BRI1-KD-catalyzed reactions. As shown in Figure 3D, all the mutants had similar catalytic activity towards BSK3-KD substrate. In contrast, a dramatic reduction of BRI1-KD activity for BKI1-Cter in the range of 25-50-fold was observed upon replacement of three crucial hydrophobic residues with an Asp (BRI1-KD ${ }^{\mathrm{I1108D}}$, BRI1-KD ${ }^{\mathrm{L} 1128 \mathrm{D}}$ and BRI1-KD ${ }^{\mathrm{V} 1135 \mathrm{D}}$ ). Unexpectedly, mutating Lys1132 to charged Asp had little effect on enzyme activity, suggesting that this basic residue is not important for BKI1 targeting compared to the hydrophobic residues. BKI1-Cter specifically binds to BRI1-KD but not the KD of the coreceptor BAK1 in vitro $[14,15]$. We aligned the $\mathrm{C}$-lobes of the $\mathrm{KDs}$ from the crystal structures of BAK1-KD and BRI1-KD-BIM complex as shown in Figure 3E and 3F. One of the few differences between BRI1-KD and BAK1-KD in the BIM-binding region is the presence of Ile1125/Leu1128 in BRI1-KD, which correspond to Glu533/Glu536 in BAK1-KD. The substitution of the two hydrophobic residues with negatively charged residues may completely disrupt the hydrophobic environment required for BKI1 binding on BAK1. In addition, the small residue Ala1104 in BRI1 is replaced by Leu513 with large side chain in BAK1. When we modeled BIM peptide onto the BAK1 $\mathrm{KD}$ by using the structure of BRI1-KD-BIM complex as a guide, Leu513 in BAK1 collided with the middle portion of the BIM helix. The Cter tail of BAK1-KD (residues 566-573) is ordered and positioned on the top of helix $\alpha \mathrm{G}$, where the bulky indole ring of $\operatorname{Trp} 572$ would sterically clash with the hypothetically bound BIM peptide. Together, the acidic patch and two bulky side chains in 
the BAK1-KD would impede the binding of the BIM peptide.

BRI1-BKI1 interaction is necessary to inhibit BRI1 signaling in planta

A key step in BRI1 activation is the dissociation of BKI1 from the plasma membrane (PM), and the phosphorylation of BKI1 by BRI1 plays critical roles in this process $[14,15,19]$. To explore the biological relevance of the interaction of BKI1 with BRI1, transgenic plants expressing the interface-mutated BKI1-GFP were generated. We found that none of the transgenic seedlings showed a phenotype similar to BKII overexpression lines. As shown in Figure 4A, overexpression of the wild-type $B K I 1$ resulted in dwarf plants that had a smaller rosette than the untransformed plants. In comparison with the wild-type $B K I 1, B K I 1^{L 3 I I D}$ overexpression resulted in a semidwarf phenotype, but overexpression of the other six mutants defective for interaction with BRI1 failed to produce a dwarf phenotype, despite these proteins being expressed at levels similar to wild-type BKI1 (Figure 4B). Interestingly, BKI1 ${ }^{Q 316 E}$ overexpression resulted in severe dwarf plants that resembled the phenotype of transgenic plants with overexpression of myristoylated BKI1 [14]. Indeed, Q316E substitution in BKI1-Cter increased its interaction with BRI1-KD $\left(K_{\mathrm{m}}\right.$ $=2.34 \pm 0.44 \mu \mathrm{M}$ for BKI1-Cter ${ }^{\mathrm{Q} 16 \mathrm{E}} v s K_{\mathrm{m}}=4.28 \pm 0.73$ $\mu \mathrm{M}$ for wild-type BKI1-Cter).

We also examined the subcellular distribution of BKI1 mutant forms in plants (Figure 4C). Like wildtype BKI1-GFP, BKI1 ${ }^{\mathrm{Q} 316 \mathrm{E}}$-GFP was localized to both the plasma membrane (PM) and the cytosol in root-tip cells, and BRs induced the dissociation of this mutant from the $\mathrm{PM}$, as indicated by the diminished discrete fluorescent signal on the cell surface. In contrast, BRs did not cause complete dissociation of other BKI1-GFP mutants from the PM. The localizations of the BKI1-GFP mutants are consistent with their phosphorylation efficiencies by BRI1, further confirming that release of BKI1 from the PM is triggered by multiple phosphorylation. Note that the M308D mutant appears to have some internalized BKI1, more than the partial loss-of-function mutant L311D, suggesting that the phenotype of the transgenic plant is not always correlated with BKI1 localization. Similar result has been reported by Jaillais et al. [15]. They found that although the BKI1 ${ }^{\mathrm{C} 322 \mathrm{~A}}$ transgenic plant

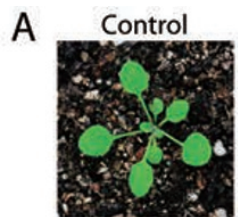

BKI1 ${ }^{\text {Q316E }}$

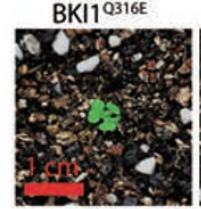

C BKI1
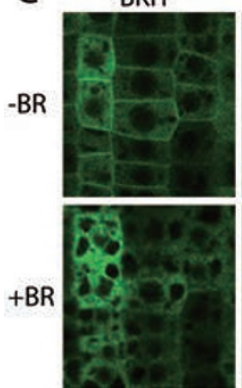
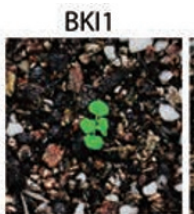

BKI1 ${ }^{A 318 D}$

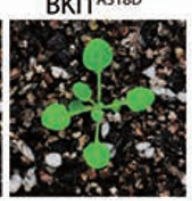

BKI1 ${ }^{\text {M308D }}$

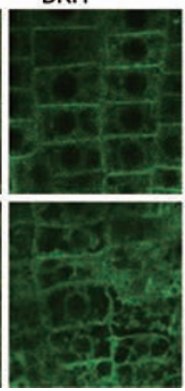

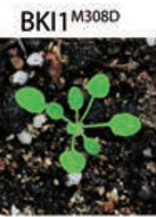

BKI1319A

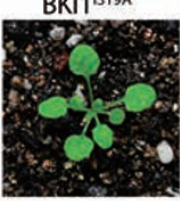

BKI1 ${ }^{13110}$

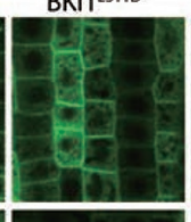

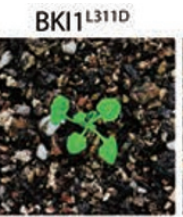

BKI1 ${ }^{\text {MAA }}$
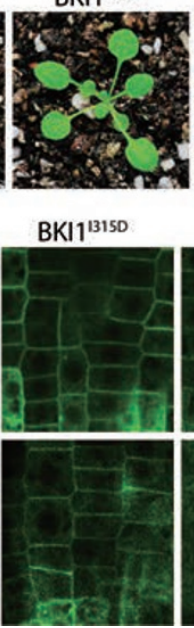

BKI11315D

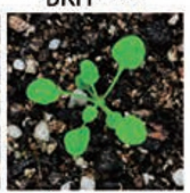

BKI1 ᄂᄂ

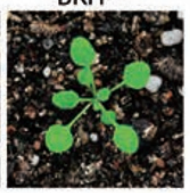

BKI10316E

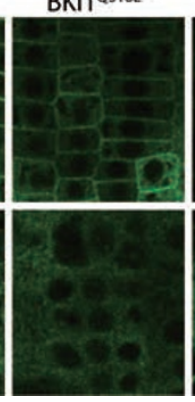

$B$
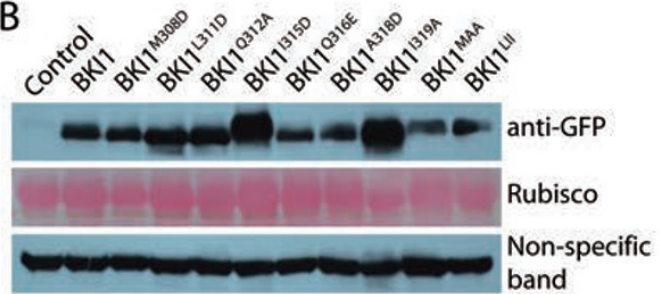
band
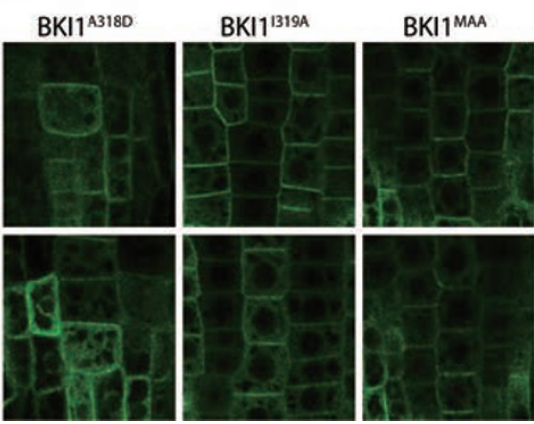

BKI1

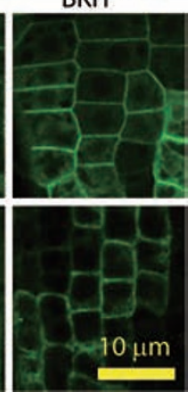

Figure 4 BKI1 mutants defective in BRI1 interaction lose their inhibitory activity in plants. (A) Phenotypes of plants expressing empty vector and various BKI1-GFP in the Columbia (Col-0) Arabidopsis. Scale bar, $1 \mathrm{~cm}$. For each construct, 30 transgenic lines with comparable levels of BKI1 expression were selected for further phenotypic analysis. The rosette radius of the plants were measured and the phenotypes in each group are consistent (Standard deviations for all data sets in each group were less than $10 \%$ of the mean). (B) Protein levels of various BKI1-GFP in transgenic plants. Ponceau-S-stained Rubisco large subunit served as a loading control. (C) Localization and membrane dissociation upon $1 \mu \mathrm{M}$ epi-BL treatment of various BKI1-GFP in root tip cells of 4-day-old seedlings. Scale bar $10 \mu \mathrm{m}$. 
showed normal growth as the Col-0, it had abundant internalized BKI1 ${ }^{\mathrm{C} 322 \mathrm{~A}}$.

$B K I 1$ binding to BRII inhibits the reciprocal transphosphorylation of BRII and BAK1

The PM-localized BRI1 is quiescent in the absence of steroids, largely due to a negative regulator, BKI1 [14, $15,19]$. BKI1 could control BRI1 activity by two possible mechanisms: (1) directly inhibit the intrinsic kinase activity of the activated BRI1 by blocking its active site, or (2) inhibit the activation process of the inactive BRI1. To distinguish these mechanisms, we first measured the binding affinities of the unphosphorylated and phosphorylated BKI1-Cter (pBKI1-Cter) with BRI1-CD by isothermal titration calorimetry (ITC; Figure 5A). The phosphorylated BKI1 has lower affinity than dephosphorylated BKI1 to BRI1 KD $\left(K_{\mathrm{d}}=3.95 \mu \mathrm{M} v s 1.61 \mu \mathrm{M}\right)$, which is plausible as phosphorylated BKI1 leaves PM and BRI1 in the presence of BRs. Furthermore, Wang et al. [16] have shown that the 14-3-3 proteins can interact with the phosphorylated BKI1 through a motif that contains the two phosphorylation sites to release inhibition of BRI1 by BKI1.

Because pBKI1-Cter is a product of the BRI1-CD-cat-
A BKI1-Cter w. BRI1-CD

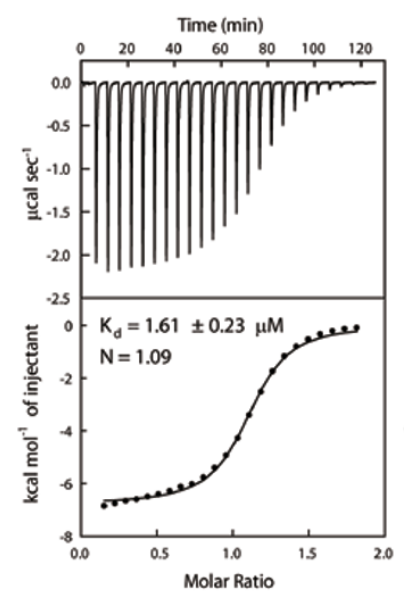

D

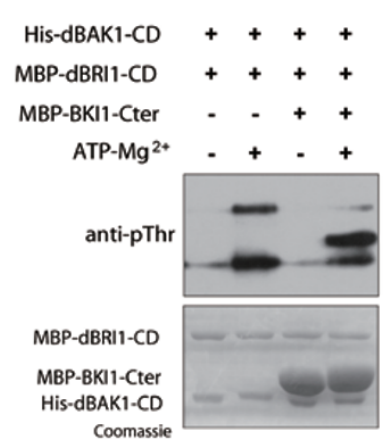

pBKI1-Cter w. BRI1-CD

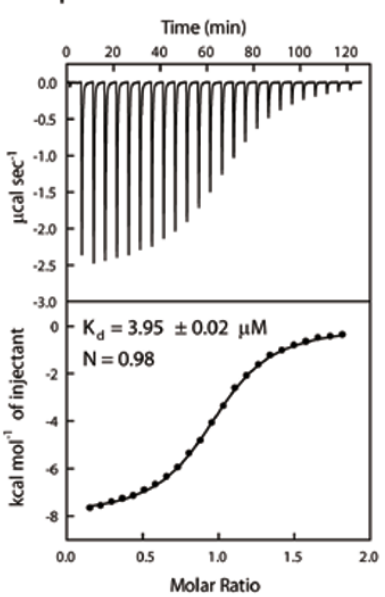

E

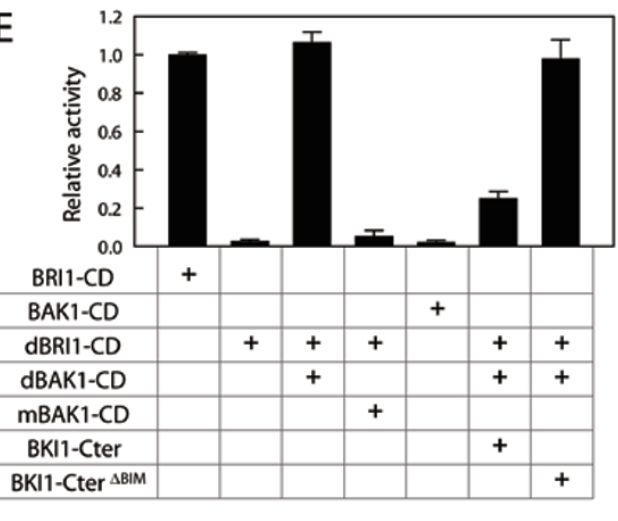

B

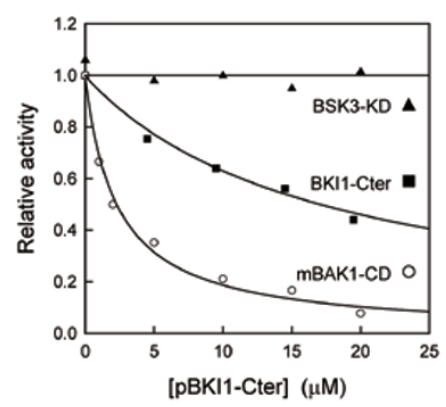

C

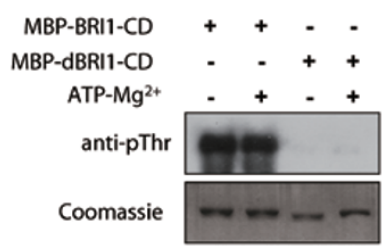

His-BAK1-CD $++-\cdot$

His-dBAK1-CD $\quad-\quad++$

ATP-Mg
anti-pThr

Coomassie

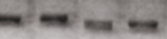

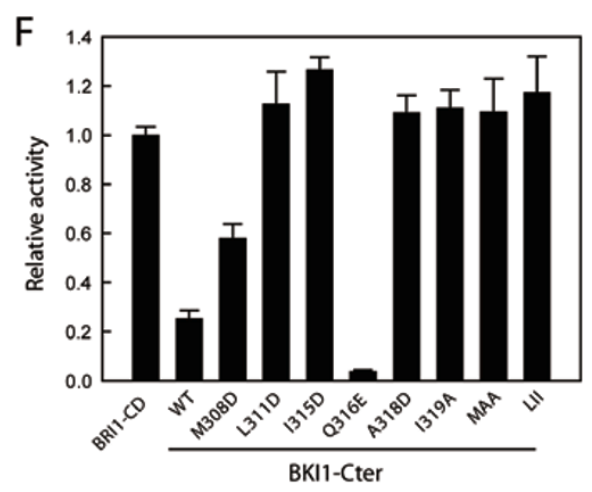

Figure 5 BKI1 inhibits transphosphorylation of BRI1 and BAK1. (A) Thermodynamic analysis of the interaction between BKI1Cter or pBKI1-Cter and BRI1-CD by ITC. The top panels show heat release during titration, and the bottom panels show the corresponding integrated heat of binding. (B) Effects of pBKI1-Cter on the BRI1-catalyzed phosphorylation reactions. The relative activity of $B R I 1-C D$ towards substrates $B S K 3-K D, B K I 1-C t e r$ and $m B A K 1-C D$ were measured in the presence of $p B-$ KI1-Cter. Reaction was initiated by adding $50 \mathrm{nM}$ BRI1-CD to the kinase reaction buffer containing $5 \mu \mathrm{M}$ substrate (BSK3-KD, $B K I 1-C t e r$ or mBAK1-CD) in the presence of different concentrations of pBKI1-Cter. The initial velocities of BSK3-KD, BKI1Cter and mBAK1-CD phosphorylation were determined. (C) Autophosphorylation of various BRI1-CD and BAK1-CD proteins analyzed by western blotting. BRI1-CD or BAK1-CD purified from E. coli was incubated with GST-PP2C $\alpha$ in vitro to produce the dephosphorylated BRI1-CD (dBRI1-CD) and the dephosphorylated BAK1-CD (dBAK1-CD). The dephosphorylated proteins were further purified to remove the phosphatase. (D and E) Effects of BKI1-Cter on transphosphorylation of dephosphorylated BRI1-CD and BAK1-CD analyzed by western blotting (D) and enzyme kinetic assays (E). (F) Inhibition of various BKI1 mutations on BRI1-BAK1 transphosphorylation (mean $\pm \mathrm{SEM}, n=3$ ). 
alyzed reaction and has high affinity for BRI1-CD, we then evaluated the ability of pBKI1-Cter to inhibit the BRI1-catalyzed reactions. As shown in Figure 5B, pBKI1-Cter efficiently inhibits the BRI1-CD-catalyzed phosphorylation of BKI1-Cter and mBAK1-CD, but has no effect on the BSK3-KD phosphorylation. This is consistent with the result obtained by in vitro GST pulldown assay that BKI1 directly inhibited the interaction between the KDs of BRI1 and BAK1 [14]. Taken together, these observations indicate that (1) BKI1-Cter recognizes BRI1-CD independent of its own phosphorylation status; (2) the binding of BKI1-Cter and BSK3-KD is not mutually exclusive, suggesting that BKI1 and BSK3 interact with different regions of BRI1.

Next, we performed two complementary experiments to further examine whether BKI1 affects the activation process of the inactive BRI1. Bacterially expressed kinases are often associated with high levels of autophosphorylation that is caused by the rapid overexpression of the kinases in a strong expression system, leading to phosphorylation of proteins before or during protein folding. Therefore, phosphorylation events during expression may occur at non-physiological sites that are not accessible as substrate sites in folded proteins [30]. To investigate whether the observed hyper-phosphorylation of BRI1-CD and BAK1-CD is non-physiological or not, in vitro autophosphorylation experiments were performed on BRI1-CD and BAK1-CD, starting with non-phosphorylated proteins. To produce the dephosphorylated BRI1-CD and the dephosphorylated BAK1CD, BRI1-CD or BAK1-CD expressed and purified from E. coli was incubated with GST-PP2C $\alpha$ at a 20:1 ratio for $1 \mathrm{~h}$ at $25^{\circ} \mathrm{C}$ in the buffer containing $10 \mathrm{mM} \mathrm{MgCl}$ and $100 \mathrm{mM} \mathrm{NaCl}$. After incubation, GST-PP2C $\alpha$ was removed by GST columns. The dephosphorylated proteins were further purified by gel filtration chromatography. The dephosphorylated form of BRI1-CD $(2 \mu \mathrm{M})$ was incubated with ATP- $\mathrm{Mg}^{2+}(1 \mathrm{mM}$ ATP and $10 \mathrm{mM}$ $\mathrm{Mg}^{2+}$ ) either in isolation or together with the dephosphorylated form of BAK1-CD $(2 \mu \mathrm{M})$ for $1 \mathrm{~h}$, followed by SDS-PAGE. As shown in Figure 5C, neither dBRI1-CD nor dBAK1-CD alone is able to undergo ATP-initiated autophosphorylation. However, when the dephosphorylated forms of BRI1-CD and BAK1-CD were mixed together in the presence of ATP- $\mathrm{Mg}^{2+}$, both of them were phosphorylated. The presence of the BKI1-Cter greatly decelerated the transphosphorylation of BRI1-CD and BAK1-CD (Figure 5D). These results seem to be inconsistent with those obtained by other groups. Oh et al. [21] first proposed that bacterially expressed BRI1 alone can undergo an intramolecular autophosphorylation in vitro, while Wang et al. [13] proposed that BRI1 autophos- phorylation was intermolecular [21]. One of the primary differences between our and other studies is that bacterially expressed BRI1 used in their studies were active and phosphorylated at multiple sites, while we used dephosphorylated BRI1 for autophosphorylation assay. Thus, our studies demonstrated that the initiation of BRI1 autophosphorylation requires the presence of BAK1.

We also performed in vitro kinase assays to examine the BRI1-CD activity with BSK3-KD substrate during the reciprocal phosphorylation of these two KDs. Under the same experimental conditions, the activation of BRI1-CD is well correlated with its phosphorylation level (Figure 5E). In addition, all the BKI1 mutants defective for interaction with BRI1 impaired inhibition for the BAK1-mediated BRI1 activation (Figure 5F). These results indicated that (1) the initial activation of either receptor kinase after hetero-dimerization is through the reciprocal transphosphorylation rather than cis-autophosphorylation; (2) binding of BKI1 to BRI1-CD blocks the interaction between the BAK1 and BRI1 KDs [14], and prevents the reciprocal transphosphorylation.

\section{Discussion}

In this study, we have determined the structure of the BRI1 KD in complex with a docking peptide derived from BKI1. While the current manuscript was under review, crystal structures of BRI1 KD in isolation and in complex with adenine nucleotides (PDB codes: 4OA2, 4OA6, 4OA9, 4OAB and 4OAC) were reported [31]. These five structures are almost identical and readily superimposable to our BRI1 KD with overall rmsd of $<0.5 \AA$ over $\sim 220 \mathrm{C} \alpha$ atoms (Figure 6A and Supplementary information, Figure S3A). In our structure, the non-hydrolyzable ATP analogue AMP-PNP adopts a catalytically incompetent configuration, similar to that observed in the BRI1-KD-ATP complex structure (4OAB; Supplementary information, Figure S3B and S3C). The $\gamma$-phosphate rotated outwards away from the catalytic Asp1009, different from its active conformation in the BRI1-KD/AMP-PNP/Mn ${ }^{2+}$ complex structure (4OA9). On close inspection, however, we found significant discrepancies in the region of residues 1 087-1 124, including helices $\alpha \mathrm{G}, \alpha \mathrm{G}^{\prime}, \alpha \mathrm{G}^{\prime}$ " and their flanking loops (termed the $\alpha \mathrm{G}$ region) while superimposing our BRI1-KD/ AMP-PNP/BIM complex to their high-resolution representative (4OAC) (Figure 6B and 6C). The amino acid sequence of this $\alpha \mathrm{G}$ region in their BRI1-KD structure is off-registered by up to 7 amino acids relative to our structure. As a consequence, key residues for interaction with the BKI1-BIM peptide, such as Val1100, Lys1101, Ala1104 and Ile1108, are "frame shift" by approximately 
A

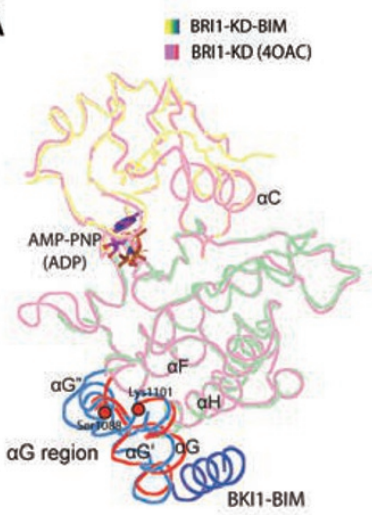

B BRI1-KD-BIM

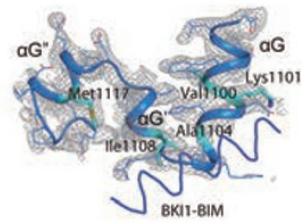

C BRI1-KD (4OAC)

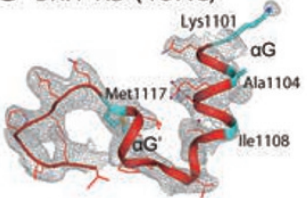

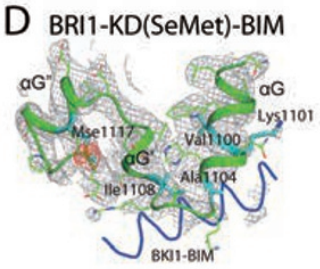

E BRI1-KD(rebuilt)

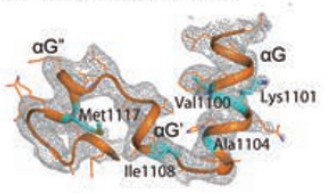

$\mathrm{F}$

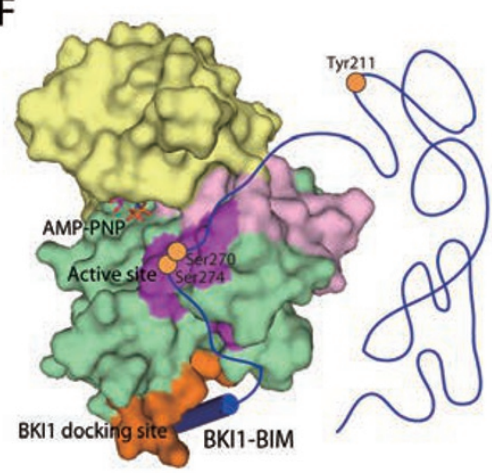

G

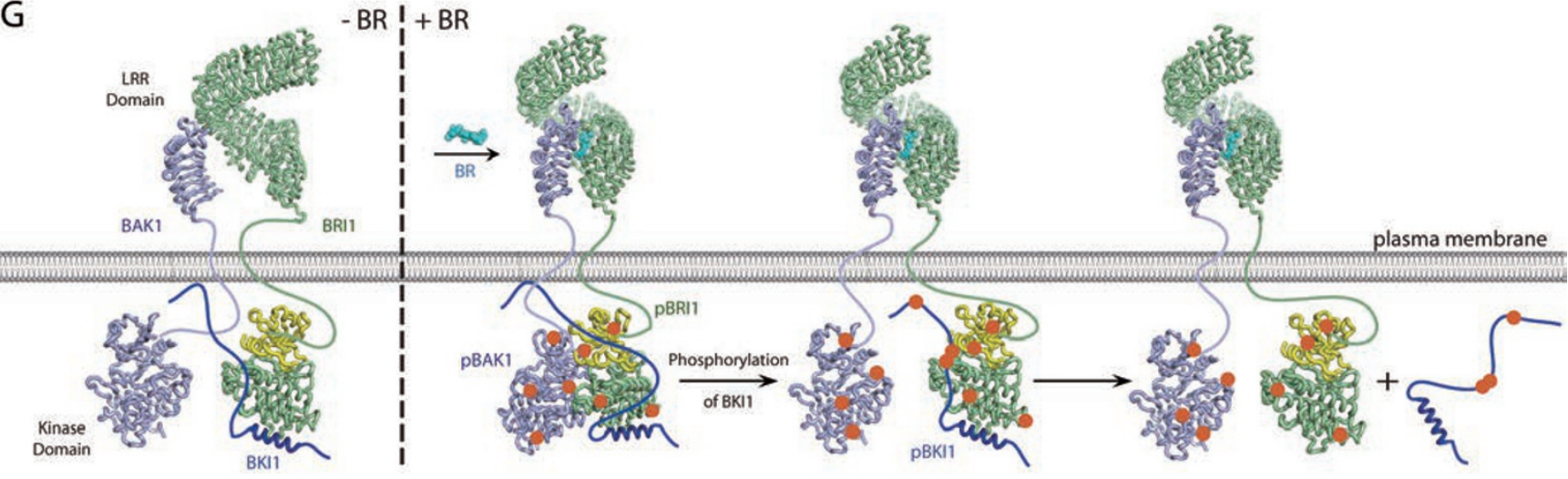

Figure 6 Model for early events of BR signaling. (A) Superposition of our BRI1-KD-BIM complex with the reported BRI1-KD structure (4OAC). The $\alpha \mathrm{G}$ regions of residues $1087-1124$ in our and reported structures are highlighted in marine blue and red, respectively. (B-E) Close-up views of the $\alpha G$ regions in our native (B) and SeMet-labeled (D) BRI1-KD-BIM complexes and in the reported $(\mathbf{C})$ and rebuilt $(E)$ BRI1-KD structures. The $2 F_{0}-F_{c}$ electron density maps (contoured at $0.8 \sigma$ ) are shown in gray mesh. In panel D, the anomalous difference Fourier map around the seleno-methionine Mse1117 (contoured at 3.0 $\sigma)$ is shown in red mesh. Key residues for BKI1 interaction are highlighted in cyan sticks. (F) Interaction model of BRI1 and BKI1. BRI1-KD is shown in surface representation, and the phosphorylation sites on BKI1 are shown as orange dots. (G) Working model for the regulation of BR signaling receptor complexes. Without BR, BKI1 competitively inhibits the transphosphorylation of BAK1 and BRI1. BR-induced heterodimerization of BRI1 and BAK1 LRR domains brings their cytoplasmic regions in the right orientation to remove BKI1 inhibition and trigger transphosphorylation of two kinase domains. The activated BRI1 then phosphorylates BKI1, which accelerates its membrane dissociation. The BRI1-BAK1 complex is fully activated. Notably, the initiation of BR signaling may also involve a small amount of preassembled BRI1/BAK1 heterodimers or BRI1 homodimers [39, 40]. See also Supplementary information, Figure S3 and Table S2.

two helix turns. In their reported structure, the preceding loop of helix $\alpha \mathrm{G}$ (1 089-1 100) was disordered (Figure $6 \mathrm{~A})$. Although the electron density for the rest of the $\alpha \mathrm{G}$ region is largely continuous over the main chain, there is insufficient density to justify building side-chains (Figure 6E and Supplementary information, Table S2). One obvious question is whether the observed differences in the $\alpha \mathrm{G}$ region are modulated by BKI1 binding. To address this issue, we first used selenium anomalous signals to help validate the atomic model of our BRI1KD-BIM complex (Supplementary information, Figure S3D and Table 1). Notably, residue Met1117 is indubita- bly located on helix $\alpha G$ " (Figure 6D), indicating that the assignment of the $\alpha \mathrm{G}$ region in our structure is reliable. In addition, BRI1 and BAK1 share approximately $70 \%$ sequence similarity in the $\mathrm{KD}$ and $60 \%$ similarity in the $\alpha \mathrm{G}$ region, making direct comparison between these two proteins relevant. There are two reported structures of the BAK1-KD, one in isolation (3UIM) and the other in complex with AvrPtoB (3TL8) [26, 32]. The $\alpha \mathrm{G}$ region of BAK1-KD mediates the tight interaction with the effector protein AvrProB. Structural analyses and sequence alignment reveal that both BAK1-KDs adopt a conformation in the $\alpha \mathrm{G}$ region very similar to that in our BRI1- 
KD-BIM structure (Supplementary information, Figure S3A). Therefore, we believe that the conformation of the $\alpha \mathrm{G}$ region in BRI1-KD is independent of BKI1 binding.

Structural and mutagenesis studies showed that the $\alpha \mathrm{G}$ region of BRI1 is required for recognition of the BKI1BIM peptide, and shed light on the importance of this interface for BKI1-mediated inhibition. BKI1 has three phosphorylation sites that are 30-100 residues away from the BIM sequence $[15,19]$. These sites are predicted to reside in the disordered polypeptide regions, suggesting that these regions are dynamic when bound to BRI1 and that interactions at the BIM motif tether the substrate to BRI1 allowing the phosphosite(s) to encounter the catalytic site and be phosphorylated (Figure 6F). Several mechanical events have been shown to contribute to the ligand-induced activation of BRI1. These include ligand-induced conformational change in a preformed homodimer or receptor homodimerization and autophosphorylation of BRI1 KD, phosphorylation and disassociation of BKI1 from the PM, and association and transphosphorylation with the BAK1 coreceptor kinase [17, 33]. In order to determine the exact sequence and causal relationship of these events, we investigated the detailed process of BRI1/BAK1 activation with dephosphorylated BRI1-CD and BAK1-CD. Current and previous studies provide several lines of evidence to strongly support that phosphorylation of BRI1 by BAK1 is indispensable to the early events of the BR signaling pathway. The evidence for this may be summarized as follows:

(1) Bacterially expressed KDs of BRI1 and BAK1 are phosphorylated at multiple sites when purified from $E$. coli, while the corresponding kinase-dead mutants, mBRI1-CD and mBAK1-CD, were completely unphosphorylated (Figure 1). BRI1-CD, but not BAK1-CD, can phosphorylate mBAK1. In contrast, BAK1-CD, but not BRI1-CD, can phosphorylate mBRI1-CD.

(2) Although bacterially expressed BRI1-CD and BAK-CD are catalytically active, neither dephosphorylated BRI1-CD nor BAK 1-CD is able to undergo activating autophosphorylation when incubated alone with ATP- $\mathrm{Mg}^{2+}$ in vitro. In contrast, both of them can be phosphorylated and activated when they were incubated together in the presence of ATP- $\mathrm{Mg}^{2+}$ (Figure 5). These results suggest that the reciprocal transphosphorylation on the cytoplasmic domains of BRI1 and BAK1 in the complex is a prerequisite for the full activation of the receptor kinase.

(3) In yeast, BRI1-BAK1 interaction activates both receptor kinases. Little phosphorylation was detected for BRI1 when it was expressed alone, however, both BRI1 and BAK1 were phosphorylated when they were coexpressed in yeast cells. Yet, no phosphorylation on either receptor kinase was detected when one of the receptor kinases was mutated. These results imply that the full activation of either receptor kinase after heterodimerization is through transphosphorylation rather than cis-phosphorylation [5].

(4) BAK1 is a conserved member of the SERK subfamily of LRR-RLKs [34]. Genetic and biochemical studies showed that four out of five members of the SERK subfamily (SERK1 to SERK4) may play functionally redundant roles in BR signaling. Detailed analysis indicated that, without BAK1 and its functionally redundant proteins, BR signaling is completely disrupted, largely because BRI1 has lost its ability to activate downstream components [35]. This study provided clear genetic and biochemical evidence that BRI1 cannot initiate BR signaling without BAK1 and its homologues. The structure of BRI1-BL-SERK1 complex revealed that the BRI1-LRR and SERK1-LRR form heterodimer in a BR-dependent manner, suggesting that BRs act as "molecular glues" for receptor-coreceptor interaction [36].

Based on the results presented in this study and the previous knowledge on BR signaling, we propose the following model for the activation of a membrane receptor upon BR binding (Figure 6G). In the absence of steroid ligand, the BR-binding site (a region that includes $\sim 70$ amino acid 'island' domain) in the BRI1 extracellular domain is largely disordered [37, 38], and the cytoplasmic KD of BRI1 associates tightly with BKI1. The BRI1BKI1 complex limits the interaction of BRI1 with BAK1 $\mathrm{KD}$ and prevents the activation of BRI1. Hormone binding induces a conformational rearrangement and fixing of the island domain. The steroid ligand and the now fully ordered island domain together form a docking platform that allows for the steroid-induced binding of the small coreceptor kinase BAK1 $[33,36]$. Physical interaction between the BRI1 and BAK1 extracellular domains brings their cytoplasmic KDs closer together, and makes them competent to transphosphorylate each other. BAK1 has a low basal kinase activity that is responsible for the BRI1 phosphorylation upon heterodimerization. The major function of BRI1-BAK1 complex is to increase the "effective concentration" of the phosphorylation sites of the BRI1 in the vicinity of the active site of BAK1, and hence enhances the catalytic efficiency for BRI1 phosphorylation. The BAK1-activated BRI1 KD is able to rapidly phosphorylate multiple substrates, such as BAK1, BKI1 and BSK3, for activating downstream signaling responses $[4,5,14,18]$. The phosphorylated BKI1 dissociates from PM and BRI1, and then binds to 14-33 proteins in cytosol to free BES1/BZR1. The released BES1/BZR1 accumulates in the nucleus, leading to BR-responsive gene expression $[15,19]$. The BRI1-acti- 
vated BAK1, in turn, transphosphorylates BRI1 rapidly, leading to enhanced BR signaling and increased plant growth.

Recently, Bücherl et al. [39] investigated the spatial correlation between BRI1 and BAK1 in the PM of live Arabidopsis root epidermal cells using comparative colocalization analysis and fluorescence lifetime imaging microscopy. They showed that approximately $7 \%$ of the BRI1 PM pool constitutively heterodimerizes with BAK1 in live root cells and activation of BR signaling by exogenous ligand application resulted in an about $50 \%$ increase of receptor heterodimerization in the PM. Our model can be extended to include the contribution of the BR-independent BRI1-BAK1 heterodimerization equilibrium (Supplementary information, Figure S3E). This model may also expand beyond BRI1/BAK1 pair of receptors due to the multi-functions of BAK1 and its homologues. A flagellin-induced FLS2-BAK1 complex activates and initiates plant defense signaling probably in a similar manner to BRI1-BAK1. Moreover, BAK1 and its homologues also function as coreceptors for various ligand-binding LRR-RLKs, such as BIR1 and PEPR1, to regulate cell death and immunity-related signaling pathways [34].

\section{Materials and Methods}

\section{Constructs, mutagenesis and protein purification}

Fragments of BRI1, BAK1, BKI1 and BSK3 were overexpressed in BL21(DE3) cells as His-tagged and/or MBP-fusion proteins. Mutations were generated by an overlap PCR procedure. All proteins were purified over Ni-NTA or amylase resin, ion-exchange and gel filtration chromatography.

\section{Kinase assays for BRII}

The kinase activity of BRI1 was determined with BSK3-KD or BKI1-Cter as substrates using a coupled spectrophotometric assay. The kinetic parameters were obtained by fitting the experimental data to the Michaelis-Menten equation.

\section{Crystallography}

Crystals of the native or SeMet-labeled BRI1-KD-BIM were grown by mixing the protein complex with an equal volume of reservoir solution containing 0.1 M HEPES (pH 7.5), 20\% PEG 3350 and $0.2 \mathrm{M} \mathrm{Li}$ Citrate. The diffraction data sets were collected at beamline BL17U at the Shanghai Synchrotron Radiation Facility, and the structure was solved by molecular replacement. The data processing and refinement statistics were summarized in Table 1.

\section{Confocal imaging of transgenic plants}

Floral dip method was used to generate transgenic lines of wild-type and mutated BKI1-GFP in the Columbia background. Confocal microscopy images of various BKI1-GFP in $\mathrm{T}_{2}$ seedlings were taken with a Leica SP8 microscope using $488 \mathrm{~nm}$ excitation. For exogenous BR treatment, the root tips were first observed without and then treated with $1 \mu \mathrm{M}$ epi-brassinolide for $10 \mathrm{~min}$ on slides.

More methods and details were described in Supplementary information, Data S1.

\section{Accession numbers}

The atomic coordinates of the native and SeMet-labeled BRI1KD-BIM complex have been deposited in the Protein Data Bank under accession codes $4 \mathrm{OH} 4$ and 4Q5J, respectively.

\section{Acknowledgments}

We thank JH He and other staff members of beamline BL17U at SSRF for assistance in data collection. ZXW is supported by the National Basic Research Program of China (2011CB910800) and the National Natural Science Foundation of China (31270848). XW is supported by the National Natural Science Foundation of China (91117005, 30925020) and the National Basic Research Program of China (2012CB114300).

\section{References}

1 Thiel KW, Carpenter G, Flickinger MC. Cell-surface receptors: structure, activation, and signaling. Encyclopedia of Industrial Biotechnology: John Wiley \& Sons, Inc. 2010:1-9.

2 Shiu SH, Karlowski WM, Pan R, Tzeng YH, Mayer KF, Li WH. Comparative analysis of the receptor-like kinase family in Arabidopsis and rice. Plant Cell 2004; 16:1220-1234.

3 Li J, Chory J. A putative leucine-rich repeat receptor kinase involved in brassinosteroid signal transduction. Cell 1997; 90:929-938.

4 Li J, Wen J, Lease KA, Doke JT, Tax FE, Walker JC. BAK1, an Arabidopsis LRR receptor-like protein kinase, interacts with BRI1 and modulates brassinosteroid signaling. Cell 2002; 110:213-222.

5 Nam KH, Li J. BRI1/BAK1, a receptor kinase pair mediating brassinosteroid signaling. Cell 2002; 110:203-212.

6 Clouse SD. Brassinosteroid signal transduction: from receptor kinase activation to transcriptional networks regulating plant development. Plant Cell 2011; 23:1219-1230.

7 Kim TW, Wang ZY. Brassinosteroid signal transduction from receptor kinases to transcription factors. Annu Rev Plant Biol 2010; 61:681-704.

8 Li J, Jin H. Regulation of brassinosteroid signaling. Trends Plant Sci 2007; 12:37-41.

9 Vert G, Nemhauser JL, Geldner N, Hong F, Chory J. Molecular mechanisms of steroid hormone signaling in plants. Annu Rev Cell Dev Biol 2005; 21:177-201.

10 Wang ZY, Seto H, Fujioka S, Yoshida S, Chory J. BRI1 is a critical component of a plasma-membrane receptor for plant steroids. Nature 2001; 410:380-383.

11 He Z, Wang ZY, Li J, et al. Perception of brassinosteroids by the extracellular domain of the receptor kinase BRI1. Science 2000; 288:2360-2363.

12 Kinoshita T, Cano-Delgado A, Seto H, et al. Binding of brassinosteroids to the extracellular domain of plant receptor kinase BRI1. Nature 2005; 433:167-171.

13 Wang X, Li X, Meisenhelder J, et al. Autoregulation and homodimerization are involved in the activation of the plant steroid receptor BRI1. Dev Cell 2005; 8:855-865. 
14 Wang X, Chory J. Brassinosteroids regulate dissociation of BKI1, a negative regulator of BRI1 signaling, from the plasma membrane. Science 2006; 313:1118-1122.

15 Jaillais Y, Hothorn M, Belkhadir Y, et al. Tyrosine phosphorylation controls brassinosteroid receptor activation by triggering membrane release of its kinase inhibitor. Genes Dev 2011; 25:232-237.

16 Wang H, Yang C, Zhang C, et al. Dual role of BKI1 and 14-3$3 \mathrm{~s}$ in brassinosteroid signaling to link receptor with transcription factors. Dev Cell 2011; 21:825-834.

17 Wang X, Kota U, He K, et al. Sequential transphosphorylation of the BRI1/BAK1 receptor kinase complex impacts early events in brassinosteroid signaling. Dev Cell 2008; 15:220235.

18 Tang W, Kim TW, Oses-Prieto JA, et al. BSKs mediate signal transduction from the receptor kinase BRI1 in Arabidopsis. Science 2008; 321:557-560.

19 Kim TW, Guan S, Burlingame AL, Wang ZY. The CDG1 kinase mediates brassinosteroid signal transduction from BRI1 receptor kinase to BSU1 phosphatase and GSK3-like kinase BIN2. Mol Cell 2011; 43:561-571.

20 Sreeramulu S, Mostizky Y, Sunitha S, et al. BSKs are partially redundant positive regulators of brassinosteroid signaling in Arabidopsis. Plant J 2013; 74:905-919.

21 Oh MH, Ray WK, Huber SC, Asara JM, Gage DA, Clouse SD. Recombinant brassinosteroid insensitive 1 receptor-like kinase autophosphorylates on serine and threonine residues and phosphorylates a conserved peptide motif in vitro. Plant Physiol 2000; 124:751-766.

22 Lochhead PA, Sibbet G, Morrice N, Cleghon V. Activation-loop autophosphorylation is mediated by a novel transitional intermediate form of DYRKs. Cell 2005; 121:925-936.

23 Roskoski R Jr. Assays of protein kinase. Methods Enzymol 1983; 99:3-6.

24 Oh MH, Wang X, Kota U, Goshe MB, Clouse SD, Huber SC. Tyrosine phosphorylation of the BRI1 receptor kinase emerges as a component of brassinosteroid signaling in Arabidopsis. Proc Natl Acad Sci USA 2009; 106:658-663.

$25 \mathrm{Oh}$ MH, Clouse SD, Huber SC. Tyrosine phosphorylation of the BRI1 receptor kinase occurs via a post-translational modification and is activated by the juxtamembrane domain. Front Plant Sci 2012; 3:175.

26 Yan L, Ma Y, Liu D, et al. Structural basis for the impact of phosphorylation on the activation of plant receptor-like kinase BAK1. Cell Res 2012; 22:1304-1308.

27 Wang X, Goshe MB, Soderblom EJ, et al. Identification and functional analysis of in vivo phosphorylation sites of the
Arabidopsis BRASSINOSTEROID-INSENSITIVE1 receptor kinase. Plant Cell 2005; 17:1685-1703.

28 Olah GA, Mitchell RD, Sosnick TR, Walsh DA, Trewhella J. Solution structure of the cAMP-dependent protein kinase catalytic subunit and its contraction upon binding the protein kinase inhibitor peptide. Biochemistry 1993; 32:3649-3657.

29 Rost B, Sander C. Prediction of protein secondary structure at better than 70\% accuracy. J Mol Biol 1993; 232:584-599.

30 Shrestha A, Hamilton G, O'Neill E, Knapp S, Elkins JM. Analysis of conditions affecting auto-phosphorylation of human kinases during expression in bacteria. Protein Expr Purif 2012; 81:136-143.

31 Bojar D, Martinez J, Santiago J, Rybin V, Bayliss R, Hothorn M. Crystal structures of the phosphorylated BRI1 kinase domain and implications for brassinosteroid signal initiation. Plant J 2014; 78:31-43.

32 Cheng W, Munkvold KR, Gao H, et al. Structural analysis of Pseudomonas syringae AvrPtoB bound to host BAK1 reveals two similar kinase-interacting domains in a type III Effector. Cell Host Microbe 2011; 10:616-626.

33 Jaillais Y, Belkhadir Y, Balsemao-Pires E, Dangl JL, Chory J. Extracellular leucine-rich repeats as a platform for receptor/ coreceptor complex formation. Proc Natl Acad Sci USA 2011; 108:8503-8507.

34 Li J. Multi-tasking of somatic embryogenesis receptor-like protein kinases. Curr Opin Plant Biol 2010; 13:509-514.

35 Gou X, Yin H, He K, et al. Genetic evidence for an indispensable role of somatic embryogenesis receptor kinases in brassinosteroid signaling. PLoS Genet 2012; 8:e1002452.

36 Santiago J, Henzler C, Hothorn M. Molecular mechanism for plant steroid receptor activation by somatic embryogenesis co-receptor kinases. Science 2013; 341:889-892.

37 Hothorn M, Belkhadir Y, Dreux M, et al. Structural basis of steroid hormone perception by the receptor kinase BRI1. $\mathrm{Na}$ ture 2011; 474:467-471.

38 She J, Han Z, Kim TW, et al. Structural insight into brassinosteroid perception by BRI1. Nature 2011; 474:472-476.

39 Bucherl CA, van Esse GW, Kruis A, et al. Visualization of BRI1 and BAK1(SERK3) membrane receptor heterooligomers during brassinosteroid signaling. Plant Physiol 2013; 162:1911-1925.

40 Hink MA, Shah K, Russinova E, de Vries SC, Visser AJ. Fluorescence fluctuation analysis of Arabidopsis thaliana somatic embryogenesis receptor-like kinase and brassinosteroid insensitive 1 receptor oligomerization. Biophys $J$ 2008; 94:1052-1062.

(Supplementary information is linked to the online version of the paper on the Cell Research website.) 